\title{
Diagnosis of solitary extramedullary plasmacytoma located in the nasopharynx in a patient with acquired angioedema
}

\author{
Marcin Czaban, Marcin Moniuszko, Maciej Klimek, Mateusz Lukaszyk, Malgorzata Rozanska, Joanna Reszec, \\ Marek Rogowski, Anna Bodzenta-Lukaszyk
}

Department of Allergology and Internal Medicine, Medical University of Bialystok, Bialystok, Poland

Adv Dermatol Allergol 2018; XXXV (6): 636-637

DOI: https://doi.org/10.5114/ada.2018.77616

Solitary extramedullary plasmacytoma (SEP) is a relatively rarely diagnosed and reported form of multiple myeloma with absent or low serum or urinary level of monoclonal immunoglobulin that most frequently presents as a soft tissue mass in the head and neck area [1, 2].

A 64-year-old Caucasian man with a history of recurrent angioedema, diabetes mellitus type 2 , hypertension and alcohol dependence syndrome was referred to the University Hospital of Bialystok due to the sudden onset of angioedema without accompanying difficulty in breathing or swallowing. On admission to the Emergency Room, he presented angioedema of the upper lip, right cheek as well as tongue edema but no hives or itch. In the Emergency Room, the patient received systemic corticosteroids and antihistamines, and he was further referred to the Department of Allergology and Internal Medicine for treatment continuation.

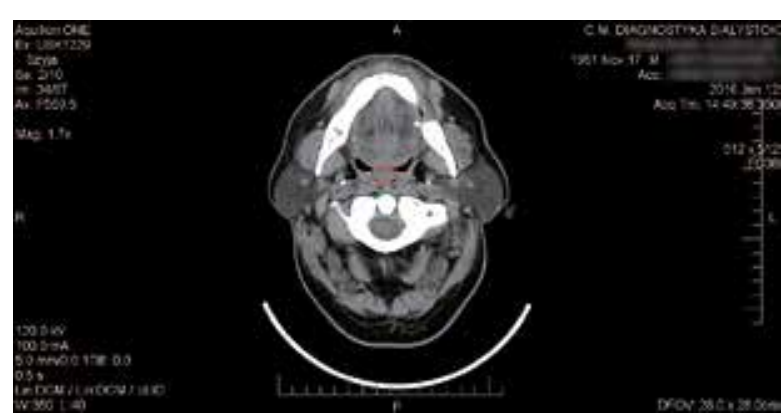

Figure 1. Plasmacytic myeloma. Non-contrast axial computed tomography scan of the neck was performed and revealed a $15 \mathrm{~mm}$ by $19 \mathrm{~mm}$ tumor in the pharynx (red circle) located between the soft palate and lower part C2 vertebra. Computed tomography scan demonstrated a circularly thickened pharynx and esophagus stenosis. Neck lymph nodes were not involved
Following admission to the clinical ward and gradual decrease in the edema size, the patient was examined by a board-certified otolaryngologist. The ENT examination revealed the presence of the oropharynx tumor covered by a fibrin layer which appeared to have originated from the nasopharynx. In concert with this notion, head and neck computed tomography showed an exophytic mass $(15 \times 19 \mathrm{~mm})$ within oropharynx heading to its lumen (Figure 1). Surprisingly, histopathological and immunohistochemical analysis of the tumor specimen revealed diagnosis of plasmacytic myeloma. In some contrast, basic analyses as well as other standard tests routinely performed in either multiple myeloma or angioedema patients did not reveal significant abnormalities. Importantly, both myelogram and trepanobiopsy did not reveal the presence of malignant plasma cells. The $\mathrm{X}$-ray survey did not reveal the presence of any osteolytic bone lesions.

After having established the diagnosis of solitary extramedullary plasmacytoma, the patient was qualified for radiotherapy to be performed in the settings of the oncological ward. Interestingly, according to the medical reports from previous hospitalization 4 years before, the presence of the unspecified lesion at the same location was also documented and referred to as putative fibroma. The patient was then recommended to have a histopathological examination, however he refused to do so. During the following 4 years, the patient did not report such changes in the clinical status as otherwise unexplained weight loss or fatigue.

This case illustrates the rare possibility of diagnosis of solitary extramedullary plasmacytoma in patients with non-specific oropharynx tumors. On the other hand, it is not that rare that patients with angioedema are being diagnosed with different comorbidities in the course of more detailed clinical evaluation. Acquired angioedema can quite frequently accompany inflammatory diseases

Address for correspondence: Marcin Moniuszko, Department of Allergology and Internal Medicine, Medical University of Bialystok, 24 A M. Sklodowskiej-Curie St, 15-276 Bialystok, Poland, phone: +48 509 138 579, e-mail: Marcin.Moniuszko@umb.edu.pl Received: 16.03.2018, accepted: 12.07.2018. 
and malignancies or, in some cases, it can even precede their further clinical onset [3]. Of malignancies, acquired angioedema is relatively common among patients with B-cell lymphocytic lymphoma [4]. To our knowledge, however, this is the first report of angioedema accompanying solitary extramedullary plasmacytoma.

Co-existence of SEP and angioedema in our patient does not necessarily indicate the causative relationship between these two disorders. Nevertheless, this case confirms several previous observations indicating that angioedema patients require a more comprehensive differential diagnosis.

\section{Conflict of interest}

The authors declare no conflict of interest.

\section{References}

1. Soutar R, Lucraft H, Jackson G, et al. Guidelines on the diagnosis and management of solitary plasmacytoma of bone and solitary extramedullary plasmacytoma. Br J Haematol 2004; 124: 717-26.

2. Ghazizadeh M, Alavi Amlashi H, Mehrparvar G. Radioresistant extramedullary plasmacytoma of the maxillary sinus: a case report and review article. Iran J Otorhinolaryngol 2015; 27: 313-8.

3. Wu MA, Perego F, Zanichelli A, et al. Angioedema phenotypes: disease expression and classification. Clin Rev Allergy Immunol 2016; 51: 162-9.

4. Cicardi M, Zingale LC, Pappalardo E, et al. Autoantibodies and lymphoproliferative diseases in acquired C1-inhibitor deficiencies. Medicine (Baltimore) 2003; 82: 274-81. 